typeset using JPSJ.sty $<$ ver.1.0b $>$

\title{
The rejuvenation effect in the two-state random energy model
}

\author{
Mitsuhiro KaWASAK团 \\ Department of Physics, Kyushu University, Fukuoka 812-8581, Japan
}

(Received November 15, 2018)

\begin{abstract}
Theoretical analyses of the random energy model with only two states and its extension with a hierarchy of only two levels show that these models reproduce out-of-equilibrium phenomena observed in experiments of glassy materials; the rejuvenation effect (the chaos effect), i.e. the abrupt jump and subsequent relaxation of the out-of-phase susceptibility as if the system rejuvenates when the temperature is lowered, and the power-law relaxation of the two-time correlation function. Our results suggest that also in an assembly of small systems with relaxation times distributed broadly some of these interesting out-of-equilibrium phenomena can be observed.
\end{abstract}

KEYWORDS: random energy model, hierarchical diffusion, rejuvenation, chaos effect, reinitialization

\section{§1. Introduction}

The free energy of disordered systems such as spin-glasses, structural glasses, polymers and proteins 1 is considered to have a very complex structure with numerous local minima. Due to this complexity, it takes very long time for a system to equilibrate and various out-of-equilibrium phenomena are observed even in macroscopic time-scales.2]

For example, in the temperature cycling experiment reported for spin-glasses, , , $_{\text {, }}$ the relaxation of the out-of-phase susceptibility $\chi^{\prime \prime}$ is measured in the following three stages. In the first stage, the sample is quenched from above $T_{g}$ down to a temperature $T$ below $T_{g}$ and is kept at this temperature for a period of $t_{1}$. Then the sample is perturbed by changing the temperature from $T$ to $T-\triangle T$ and is kept at $T-\triangle T$ for a period of $t_{2}$ (the second stage). After that, the temperature is returned to $T$ in the third stage. The effect of the perturbation is examined by comparing the perturbed data with unperturbed data in the third stage. In the case $\triangle T>0$, both data coincide except at the very beginning of the third stage. This is called the memory effect, since the sample remembers the relaxation in the first stage. On the other hand, an abrupt jump and subsequent relaxation restarted as if the sample rejuvenates are observed at the beginning of the second stage,

\footnotetext{
*E-mail: M.Kawasaki@cmt.phys.kyushu-u.ac.jp
} 
which is called the rejuvenation effect or the chaos effect since it shows that equilibrium state at $T-\triangle T$ is very different from one at $T$, i.e. chaotic dependence of equilibrium state on temperature. These memory and rejuvenation effect are observed also in a cooling and reheating experiment for spin-glasses by Jonason et al. (4)

According to the Parisi's mean-field solution of the SK model, the free energy of the spin-glass has a very complex structure with numerous local minima. From this complex free energy landscape, very slow relaxation, aging, the memory effect and the rejuvenation effect can be explained by analyzing the random energy model and its extension.6, (6)

However, some of these out-of-equilibrium phenomena are features not restricted to systems with numerous local minima, since it is well-known that the power-law relaxation can be reproduced by an assembly of small systems with very different relaxation times. Furthermore, when the energies of local minima deviate very strongly from valley to valley, equilibrium state, i.e. the Gibbs measure, is sensitive to temperature even if the number of local minima is small. Hence, it is expected that such small systems can show the rejuvenation effect due to this sensitivity of equilibrium state to temperature. The aim of this paper is to show that the rejuvenation effect is a feature not restricted to systems with numerous local minima and models with small number of local minima can reproduce the slow relaxation and the rejuvenation effect by analyzing the random energy model with only two states and its extension with hierarchy of only two layers.

The organization of this paper is the following; In section 2 we present the models analyzed in this paper. In section 3 we show that the two-time correlation function decays in power-law. In section 4 we show that the rejuvenation effect is reproduced by analyzing the out-of-phase susceptibility. Finally our conclusion and discussion about the fluctuation-dissipation theorem are presented in section 5 .

\section{$\S 2$. The models}

In this section, we describe two models analyzed in this paper; the two-state random energy model (2S-REM) and the two-layer random energy model (2L-REM).

\subsection{The two-state random energy model}

At first we explain the 2S-REM schematically shown in Fig. 1 where only two states exists and the state energies $E$ are independent random variables identically distributed as

$$
\rho(E)= \begin{cases}\frac{1}{k_{B} T_{c}} \exp \left(-E / k_{B} T_{c}\right) & \text { when } E \geq 0 \\ 0 & \text { otherwise }\end{cases}
$$

where $T_{c}$ is a parameter to express the extent of dispersion of $E$. From the Arrhenius law, the 
(a)

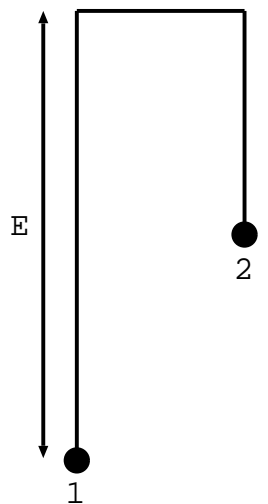

(b)

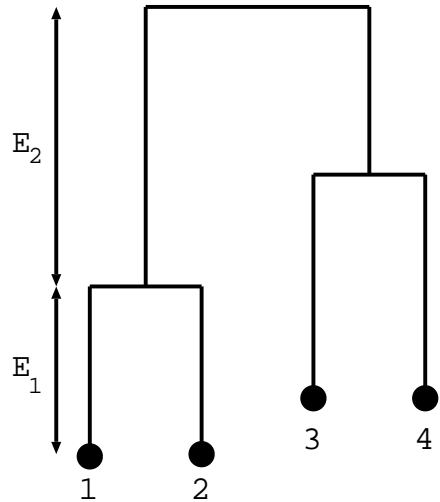

Fig. 1. The structure of the two-state random energy model (a) and the structure of the two-layer random energy model (b). The closed circles represent accessible states.

transition rate $w_{i}$ from site $i$ is related to $E$ as

$$
w_{i}=\frac{1}{\tau_{0}} \exp \left(-E / k_{B} T\right)
$$

where $\tau_{0}$ is a microscopic time scale. From eq. (2.1), it is shown that the distribution of the transition rate is given as

$$
P(w)= \begin{cases}\alpha w^{\alpha-1} & \text { when } 0<w \leq 1 \\ 0 & \text { otherwise }\end{cases}
$$

where $\alpha \equiv T / T_{c}$ and the microscopic time scale $\tau_{0}$ is set to the time unit. Referring to the two states as 1 and 2, the master equation for the probabilities $P_{i}(t)$ that the system is found at the state $i$ is written by the transition rates as

$$
\frac{d}{d t}\left(\begin{array}{c}
P_{1}(t) \\
P_{2}(t)
\end{array}\right)=\left(\begin{array}{cc}
-w_{1} & w_{2} \\
w_{1} & -w_{2}
\end{array}\right)\left(\begin{array}{l}
P_{1}(t) \\
P_{2}(t)
\end{array}\right) .
$$

Furthermore, we assume that an observable, which we call the "magnetization", takes the value $-1(+1)$ when the system is in the state $1(2)$.

\subsection{The two-layer random energy model}

The structure of the two-layer random energy model (2L-REM) is shown in Fig. 1. We assume that the system is constructed by piling up the 2S-REM two times hierarchically. Consequently, there are four states, which we refer to as 1,2,3 and 4, where we assume that the state 1(3) is directly connected to 2(4). The energy of the n-th layer counted from the bottom, $E_{n}$, is given according to the distribution $\rho\left(E_{n}\right)=\exp \left[-E_{n} / k_{B} T_{c}(n)\right] / k_{B} T_{c}(n)$ and each layer has a different 
$T_{c}(n)$ chosen so that $T_{c}(1)<T_{c}(2)$. The master equation for the probabilities that the system is found at the state $i, P_{i}(t)$, is written by the transition rates as

$$
\frac{d}{d t}\left(\begin{array}{c}
P_{1}(t) \\
P_{2}(t) \\
P_{3}(t) \\
P_{4}(t)
\end{array}\right)=\left(\begin{array}{cccc}
-w_{1}-w_{1} w_{2} & w_{1} & w_{3} w_{4} / 2 & w_{3} w_{4} / 2 \\
w_{1} & -w_{1}-w_{1} w_{2} & w_{3} w_{4} / 2 & w_{3} w_{4} / 2 \\
w_{1} w_{2} / 2 & w_{1} w_{2} / 2 & -w_{3}-w_{3} w_{4} & w_{3} \\
w_{1} w_{2} / 2 & w_{1} w_{2} / 2 & w_{3} & -w_{3}-w_{3} w_{4}
\end{array}\right)\left(\begin{array}{c}
P_{1}(t) \\
P_{2}(t) \\
P_{3}(t) \\
P_{4}(t)
\end{array}\right),
$$

where $w_{1}$ and $w_{3}$ denotes the transition rates from lower layer to upper layer and $w_{2}$ and $w_{4}$ denotes the transition rates between different branches. From the distribution of the state energy $\rho\left(E_{n}\right)$,

$w_{1}$ and $w_{3}$ distributes as $\alpha_{1} w^{\alpha_{1}-1}$ where $\alpha_{1} \equiv T / T_{c}(1)$ and $w_{2}$ and $w_{4}$ distribute as $\alpha_{2} w^{\alpha_{2}-1}$ where $\alpha_{2} \equiv T / T_{c}(2)$. Finally, we assume that the magnetization takes the values $-1-\epsilon,-1+\epsilon, 1-\epsilon$ and $1+\epsilon$ when the system is in states $1,2,3$ and 4 .

\section{$\S 3 . \quad$ The long-time behavior of the two-time correlation function}

In this section we analyze the two-time correlation function of the "magnetization" for the two models. We assume that the initial conditions are given as

$$
\begin{array}{r}
P_{1}(0)=P_{2}(0)=1 / 2 \text { for } 2 \mathrm{~S}-\mathrm{REM}, \\
P_{1}(0)=P_{2}(0)=P_{3}(0)=P_{4}(0)=1 / 4 \text { for } 2 \mathrm{~L}-\mathrm{REM}
\end{array}
$$

to see the relaxation after quench from infinitely high temperature to $T$ below $T_{c}$.

\section{$3.12 S-R E M$}

At first, we calculate the correlation function for the 2S-REM. The master equation eq. (2.4) is solved exactly and the solution is given by

$$
\begin{aligned}
& P_{1}(t)=\frac{\left(w_{1}-w_{2}\right) \exp \left[-\left(w_{1}+w_{2}\right) t\right]+2 w_{2}}{2\left(w_{1}+w_{2}\right)}, \\
& P_{1}(t)=\frac{\left(w_{2}-w_{1}\right) \exp \left[-\left(w_{1}+w_{2}\right) t\right]+2 w_{1}}{2\left(w_{1}+w_{2}\right)} .
\end{aligned}
$$

The conditional probabilities $G_{i j}(t)$ that the system is found at the state $i$ at time $t$ if the system is at the state $j$ at time 0 are given by

$$
\begin{gathered}
G_{11}(t)=\frac{w_{1} \exp \left[-\left(w_{1}+w_{2}\right) t\right]+w_{2}}{w_{1}+w_{2}}, \\
G_{12}(t)=\frac{-w_{2} \exp \left[-\left(w_{1}+w_{2}\right) t\right]+w_{2}}{w_{1}+w_{2}}, \\
G_{21}(t)=\frac{-w_{1} \exp \left[-\left(w_{1}+w_{2}\right) t\right]+w_{1}}{w_{1}+w_{2}}, \\
G_{22}(t)=\frac{w_{2} \exp \left[-\left(w_{1}+w_{2}\right) t\right]+w_{1}}{w_{1}+w_{2}} .
\end{gathered}
$$


With these quantities, the two-time correlation function $C\left(t, t^{\prime}\right)$ of the magnetization is written as

$$
C\left(t, t^{\prime}\right)=\sum_{i, j} m_{i} m_{j}<G_{i j}\left(t-t^{\prime}\right) P_{j}\left(t^{\prime}\right)>
$$

where the angle bracket denotes average over distribution of the jump rates and $m_{i}$ denotes the magnetization of the state $i$.

It is shown that when $t>>1$ (the time unit is $\left.\tau_{0}\right)$

$$
\left\langle\frac{w_{1}^{m} w_{2}^{n}}{\left(w_{1}+w_{2}\right)^{2}} \exp \left[-\left(w_{1}+w_{2}\right) t\right]\right\rangle \simeq \frac{\alpha}{2(2 \alpha+1)} \Gamma(\alpha+m) \Gamma(\alpha+n) t^{-2 \alpha},
$$

by using the asymptotic form of the incomplete gamma function $\gamma(\alpha+m, t) \simeq \Gamma(\alpha+m)-$ $t^{\alpha+m-1} \exp (-t)$. Consequently, using eq. (3.4), when $t, t^{\prime}, t-t^{\prime}>>1$ the correlation function is written as

$$
C\left(t, t^{\prime}\right) \simeq A\left[t^{-2 \alpha}-t^{\prime-2 \alpha}+2 \alpha\left(t-t^{\prime}\right)^{-2 \alpha}\right]+B
$$

where $A \equiv \alpha^{2} \Gamma(\alpha)^{2} /(2 \alpha+1), B \equiv<\left(w_{1}-w_{2}\right)^{2} /\left(w_{1}+w_{2}\right)^{2}>$. This expression of the twotime correlation function implies that the out-of-equilibrium effect, i.e. the dependence on $t^{\prime}$, persists after very long time and the equilibrium relaxation follows the power-law with the exponent $-2 T / T_{c}$.

\subsection{L-REM}

Next, we calculate the correlation function for the 2L-REM. From the symmetry of the probabilities and the conditional probabilities;

$$
\begin{array}{r}
P_{1}(t)=P_{2}(t) \equiv P_{-}(t), P_{3}(t)=P_{4}(t) \equiv P_{+}(t), \\
G_{12}(t)=G_{21}(t) \equiv G_{--}(t), G_{34}(t)=G_{43}(t) \equiv G_{++}(t), \\
G_{11}(t)=G_{22}(t) \equiv r_{--}(t), G_{33}(t)=G_{44}(t) \equiv r_{++}(t), \\
G_{31}(t)=G_{32}(t)=G_{41}(t)=G_{42}(t) \equiv G_{+-}(t), \\
G_{13}(t)=G_{14}(t)=G_{23}(t)=G_{24}(t) \equiv G_{-+}(t),
\end{array}
$$

the correlation function is rewritten in terms of the probabilities and the conditional probabilities defined above, $P_{-}(t), P_{+}(t), G_{++}(t), r_{++}(t), G_{+-}(t), G_{-+}(t)$, as

$$
\begin{aligned}
C\left(t, t^{\prime}\right)=4\left(1+\epsilon^{2}\right)<r_{--}\left(t-t^{\prime}\right) P_{-}\left(t^{\prime}\right)>+ & 4\left(1-\epsilon^{2}\right)<G_{--}\left(t-t^{\prime}\right) P_{-}\left(t^{\prime}\right)> \\
& -8<G_{-+}\left(t-t^{\prime}\right) P_{+}\left(t^{\prime}\right)>.
\end{aligned}
$$

By solving the master equation eq. (2.5), the following expressions for the probabilities and the conditional probabilities are given;

$$
P_{-}(t)=\frac{\left(w_{1} w_{2}-w_{3} w_{4}\right) \exp \left[-\left(w_{1} w_{2}+w_{3} w_{4}\right) t\right]+2 w_{3} w_{4}}{4\left(w_{1} w_{2}+w_{3} w_{4}\right)}
$$




$$
\begin{array}{r}
P_{+}(t)=\frac{-\left(w_{1} w_{2}-w_{3} w_{4}\right) \exp \left[-\left(w_{1} w_{2}+w_{3} w_{4}\right) t\right]+2 w_{1} w_{2}}{4\left(w_{1} w_{2}+w_{3} w_{4}\right)}, \\
r_{--}(t)=\frac{1}{2} \exp \left[-w_{1}\left(2+w_{2}\right) t\right]+\frac{w_{3} w_{4}+w_{1} w_{2} \exp \left[-\left(w_{1} w_{2}+w_{3} w_{4}\right) t\right]}{2\left(w_{1} w_{2}+w_{3} w_{4}\right)}, \\
G_{--}(t)=-\frac{1}{2} \exp \left[-w_{1}\left(2+w_{2}\right) t\right]+\frac{w_{3} w_{4}+w_{1} w_{2} \exp \left[-\left(w_{1} w_{2}+w_{3} w_{4}\right) t\right]}{2\left(w_{1} w_{2}+w_{3} w_{4}\right)}, \\
G_{-+}(t)=\frac{w_{3} w_{4}-w_{3} w_{4} \exp \left[-\left(w_{1} w_{2}+w_{3} w_{4}\right) t\right]}{2\left(w_{1} w_{2}+w_{3} w_{4}\right)} .
\end{array}
$$

By using the following expressions;

$$
<\exp \left(-w_{1} w_{2} t\right)>=\frac{\alpha_{1} \alpha_{2}}{\alpha_{1}-\alpha_{2}}\left[\Gamma\left(\alpha_{2}\right)-\Gamma\left(\alpha_{2}, t\right)\right] t^{-\alpha_{2}}-\frac{\alpha_{1} \alpha_{2}}{\alpha_{1}-\alpha_{2}}\left[\Gamma\left(\alpha_{1}\right)-\Gamma\left(\alpha_{1}, t\right)\right] t^{-\alpha_{1}}
$$

and for large $t, t^{\prime}$

$$
<\exp \left(-2 w_{1} t-w_{1} w_{2} t^{\prime}\right)>\simeq \alpha_{1} \alpha_{2} \Gamma\left(\alpha_{1}\right) t^{-\alpha_{1}}\left(\frac{t}{t^{\prime}}\right)^{-\alpha_{2}} \int_{0}^{t^{\prime} / t} d x \frac{x^{\alpha_{2}-1}}{(2+x)^{\alpha_{1}}},
$$

when $t, t^{\prime}, t-t^{\prime}$ are large the correlation function is consequently given as

$$
\begin{aligned}
C\left(t, t^{\prime}\right)=\frac{\alpha_{1}^{2} \alpha_{2}^{3} \Gamma\left(\alpha_{2}\right)^{2}}{\alpha_{2}\left(2 \alpha_{2}+1\right)\left(\alpha_{1}-\alpha_{2}\right)^{2}}\left[t^{-2 \alpha_{2}}-t^{\prime-2 \alpha_{2}}\right. & \left.+2 \alpha_{2}\left(t-t^{\prime}\right)^{-2 \alpha_{2}}\right]+2 \epsilon^{2} c\left(t-t^{\prime}\right)^{-\alpha_{1}} \\
& +\left\langle\frac{\left(w_{1} w_{2}-w_{3} w_{4}\right)^{2}}{\left(w_{1} w_{2}+w_{3} w_{4}\right)^{2}}\right\rangle+O\left(t^{-\alpha_{1}-\alpha_{2}}\right),
\end{aligned}
$$

where $c$ is a function of parameters, $\alpha_{1}$ and $\alpha_{2}$, defined as

$$
\begin{aligned}
c \equiv \frac{\alpha_{1}^{2} \alpha_{2}^{2} \Gamma\left(\alpha_{1}\right)}{\alpha_{2}-\alpha_{1}} \int_{0}^{\infty} d s \int_{0}^{1} d x \frac{x^{\alpha_{2}-1}}{(2+x)^{\alpha_{1}}} & \left\{\alpha_{2} s^{-\alpha_{2}-1}\left[\Gamma\left(\alpha_{2}\right)-\Gamma\left(\alpha_{2}, s\right)\right]\right. \\
& \left.-\alpha_{1} s^{-\alpha_{1}-1}\left[\Gamma\left(\alpha_{1}\right)-\Gamma\left(\alpha_{1}, s\right)\right]\right\} .
\end{aligned}
$$

This asymptotic form implies that when $2 \alpha_{2}<\alpha_{1}$, i.e. $2 T_{c}(1)<T_{c}(2)$, the dynamics in the upper layer of the hierarchy is dominant and the asymptotic form is same as that of the $2 \mathrm{~S}$ REM except for the difference of constants since the leading term of eq. (3.9) is proportional to $\left[t^{-2 \alpha_{2}}-t^{\prime-2 \alpha_{2}}+2 \alpha_{2}\left(t-t^{\prime}\right)^{-2 \alpha_{2}}\right]$. On the other hand, when $2 T_{c}(1)>T_{c}(2)$ the long-time behavior is governed by the dynamics in the lower layer, since the leading term of eq. (3.9) is proportional to $\left(t-t^{\prime}\right)^{-\alpha_{1}}$.

\section{$\S 4$. The out-of-phase susceptibility and the rejuvenation effect}

In this section we show that the $2 \mathrm{~S}-\mathrm{REM}$ and $2 \mathrm{~L}-\mathrm{REM}$ reproduce the rejuvenation effect, which is the abrupt jump and subsequent relaxation of the out-of-phase susceptibility when the temperature is lowered.

We explain the definition of the out-of-phase susceptibility. Assuming that the magnetization when the system is at the state $i$ is $m_{i}$ and $h(t)$ denotes the applied "magnetic field" at time $t$, the state energy is changed by $-m_{i} h(t)$ and the transition rate from the state $i$ is modified by the 
factor $\exp \left[-m_{i} h(t) / k_{B} T\right]$. Then the magnetization at time $t$ is given by

$$
m(t)=\sum_{i} m_{i} P_{i}(t)
$$

We assume that the applied magnetic field oscillates in time as

$$
h(t)=h e^{-i \omega t} \text { when } t \geq 0 .
$$

Then the out-of-phase susceptibility $\chi^{\prime \prime}$ is defined as the imaginary part of the susceptibility

$$
\chi(\omega, t) \equiv \lim _{h \rightarrow 0} \frac{m(t)}{h e^{-i \omega t}} .
$$

We observe the out-of-phase susceptibility defined in this way in the following temperature variation protocol; In the first stage, the sample is quenched at time 0 from infinitely high temperature to a temperature $T$ below $T_{c}$ and the oscillating magnetic field is applied at the same time. After that, the sample is kept at the temperature $T$ for a period of $t_{1}$. In the second stage, the temperature is changed from $T$ to $T-\triangle T$ and is kept at the same value for a period of $t_{2}$. Fig. 2 shows

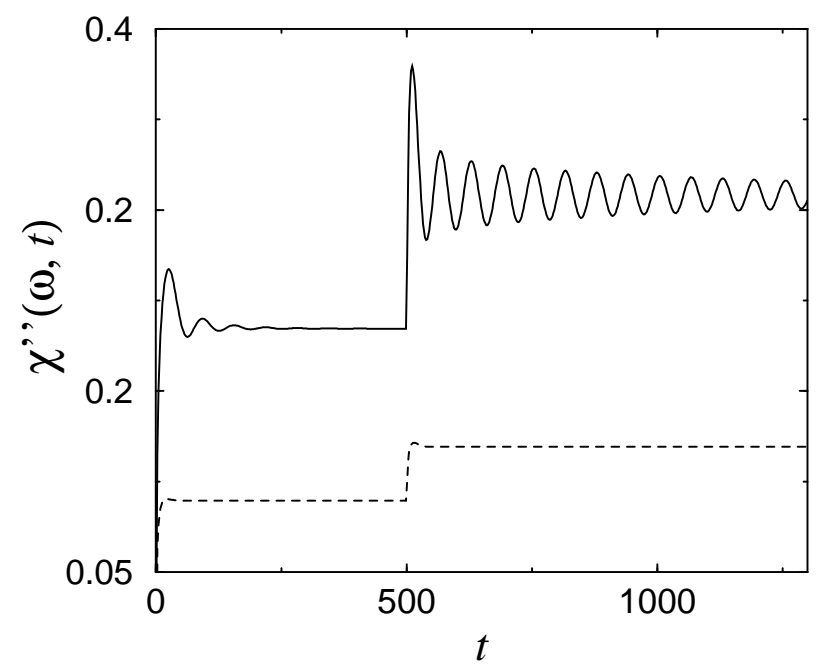

Fig. 2. The out-of-phase susceptibility of the two-state random energy model $\left(T_{c}=1\right)$ when the temperature is lowered at $t=500$ from $T=0.48$ to $T=0.28$ (the solid line) and from $T=1.0$ to $T=0.8$ (the dashed line). The frequency $\omega$ is 0.1 and the out-of-phase susceptibility is averaged over 100 samples. The abrupt jump and the subsequent relaxation, i.e. the rejuvenation effect, is clearly seen when the temperature is low.

the result of numerical calculations for $2 \mathrm{~S}-\mathrm{REM}$ when $T=0.48$ and $T=1.0, \triangle T=0.2, t_{1}=500$. We can clearly see the rejuvenation effect when $T=0.48$, i.e. the abrupt jump and the subsequent relaxation of the out-of-phase susceptibility when the temperature is changed. After the jump, $\chi^{\prime \prime}(\omega, t)$ relaxes to the new steady-state value at the temperature $T-\triangle T$ with oscillation. As 
derived in Appendix, the steady-state value when the frequency $\omega$ is low compared to $\tau_{0}$ behaves as

$$
\lim _{t \rightarrow \infty} \chi^{\prime \prime}(\omega, t) \simeq \begin{cases}\frac{1}{k_{B} T} \int_{0}^{\infty} d \tau[C(\tau)-B] \omega & \text { when } \alpha>1 / 2 \\ -\frac{1}{k_{B} T} 4 A \alpha^{2} \Gamma(-2 \alpha) \sin (\pi \alpha) \omega^{2 \alpha} & \text { when } \alpha<1 / 2,\end{cases}
$$

where the definition of the constants $A$ and $B$ are given in eq. (3.5). This result implies that the behavior of the steady-state value changes when $\alpha=1 / 2$. The qualitatively same result for the 2L-REM is given in Fig. 3.

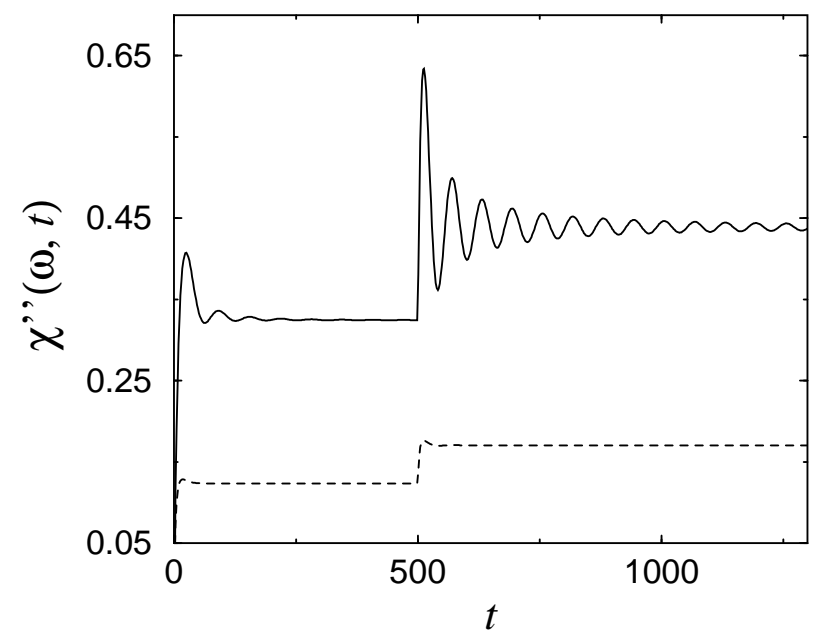

Fig. 3. The out-of-phase susceptibility of the two-layer random energy model $\left(T_{c}(1)=0.4, T_{c}(2)=1.0\right)$ when the temperature is lowered at $t=500$ from $T=0.48$ to $T=0.28$ (the solid line) and from $T=1.0$ to $T=0.8$ (the dashed line). The frequency $\omega$ is 0.1 and the out-of-phase susceptibility is averaged over 100 samples. The abrupt jump and the subsequent relaxation, i.e. the rejuvenation effect, is clearly seen when the temperature is low.

As shown in Fig. 2 and Fig. 3, the rejuvenation effect is clearly seen when the temperature is low. We show that the abrupt change becomes steep when the temperature is low or the disorder of the state energy is strong, which is the consequence of sensitivity of equilibrium state to temperature. Since the qualitative behavior of the out-of-phase susceptibility of the 2S-REM is same as that of the 2L-REM, we consider the 2S-REM for simplicity. Since the time lapse of the first stage, $t_{1}$, is sufficiently long, the system is at the steady state. By solving the master equation when $h$ is small, the probability distribution of the steady state at the temperature $T$ is derived to the order $O(h)$ as

$$
\left(\begin{array}{l}
P_{1}\left(t_{1}\right) \\
P_{2}\left(t_{1}\right)
\end{array}\right) \simeq \frac{1}{w_{1}+w_{2}}\left(\begin{array}{l}
w_{2} \\
w_{1}
\end{array}\right)+\frac{2 w_{1} w_{2} \exp \left(-i \omega t_{1}\right)}{k_{B} T\left(w_{1}+w_{2}\right)\left(i \omega-w_{1}-w_{2}\right)} h\left(\begin{array}{c}
1 \\
-1
\end{array}\right) .
$$

From this expression and the master equation eq. (2.4), the time derivative of the out-of-phase 
susceptibility immediately after the change of the temperature is given by

$$
\dot{\chi}^{\prime \prime}\left(\omega, t_{1}^{+}\right) \equiv \lim _{t \searrow t_{1}} \frac{d}{d t} \chi^{\prime \prime}(\omega, t) \simeq \frac{4}{k_{B} T} \frac{w_{1} w_{2}\left[w_{1}+w_{2}-w_{1}^{T /(T-\triangle T)}-w_{2}^{T /(T-\triangle T)}\right]}{\left(w_{1}+w_{2}\right)^{3}} \omega
$$

where $w_{1}$ and $w_{2}$ are the jump rates before the temperature is changed and we assume that the frequency $\omega$ is low. Assuming that the temperature variation $\triangle T / T$ is small, the time derivative of disorder-averaged $\chi^{\prime \prime}$ is given to the order $O(\triangle T / T)$ as

$$
<\dot{\chi}^{\prime \prime}\left(\omega, t_{1}^{+}\right)>\simeq-\frac{8 \omega}{k_{B} T}\left\langle\frac{w_{1}^{2} w_{2} \log w_{1}}{\left(w_{1}+w_{2}\right)^{3}}\right\rangle \triangle T / T .
$$

The result of numerical calculation of the expression eq. (4.7) is given in the Fig. 4. We see that the time derivative, $\left\langle\dot{\chi}^{\prime \prime}\left(\omega, t_{1}^{+}\right)>\right.$, is a monotonously increasing function of $T_{c} / T$. Since the deviation of the state energy is a monotonously increasing function of $T_{c}$, the above result implies that the abrupt change of the out-of-phase susceptibility becomes steep when the disorder of the state energy is strong or the temperature is low.

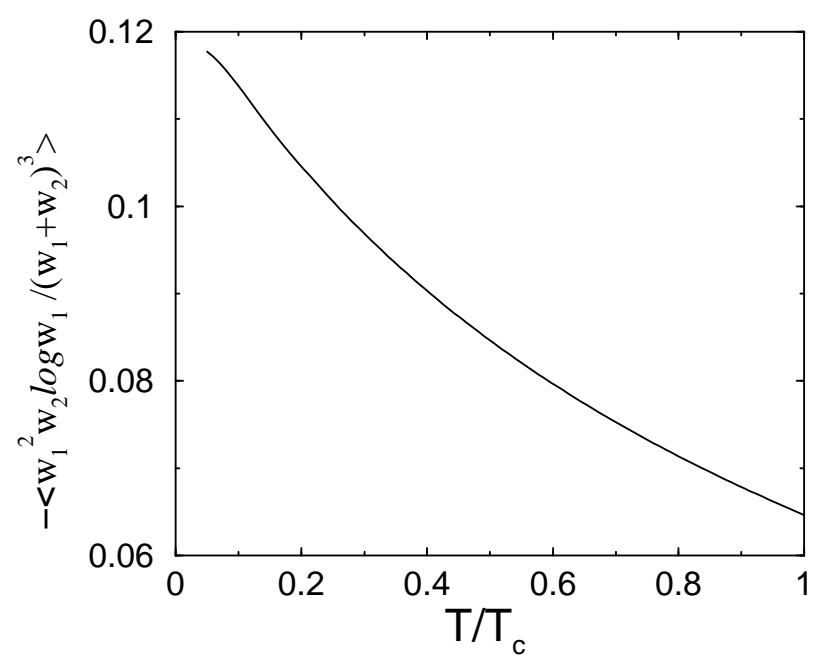

Fig. 4. The $T_{c}$-dependence of the speed of the change of the out-of-phase susceptibility when the temperature is lowered slightly. This plot shows that the speed is a increasing function of $T_{c} / T$ and the abrupt change of the out-of-phase susceptibility becomes very steep when the state energy deviates broadly or the temperature is low.

\section{§5. DISCUSSION AND CONCLUSIONS}

Summarizing our result, we have considered the out-of-equilibrium behavior of the two-state random energy model (2S-REM) and its extension with a hierarchy of two levels, i.e. 2L-REM. We have shown that these models of energy landscape with very small number of local minima can reproduce the out-of-equilibrium phenomena observed in glassy materials, especially the rejuvenation effect due to sensitivity of equilibrium state to temperature. It suggests that some of 
the interesting phenomena observed in glassy materials can be observed in an assembly of small systems with relaxation times distributed broadly even if the number of local minima is small.

We close by discussing a future issue to be solved in terms of the rugged free-energy landscape picture. Recently, it has been found that the phenomenological models based on the rugged freeenergy landscape picture can explain at least qualitatively many of significant dynamical properties of the glassy materials. 目6 However, there is an exception; violation of the fluctuation-dissipation theorem described with the violation ratio $X\left(t, t^{\prime}\right) \equiv k_{B} T R\left(t, t^{\prime}\right) / \partial_{t^{\prime}} C\left(t, t^{\prime}\right)$, where $R\left(t, t^{\prime}\right)$ denotes the response function, observed in spin-glasses and other glassy materials. 8.8) For example, it is easily shown for the models analyzed in 5,66) that the violation ratio $X\left(t, t^{\prime}\right)$ is given as $X\left(t, t^{\prime}\right)=t / t^{\prime}$ from the scaling of the correlation function $C\left(t, t^{\prime}\right)=\hat{C}\left(t / t^{\prime}\right)$ and the relation known to hold for these models $\left(k_{B} T R\left(t, t^{\prime}\right)=-\partial_{t} C\left(t, t^{\prime}\right)\right)$. (6) Hence, these models cannot reproduce the constant violation factor observed in the p-spin spherical spin-glasses and in the numerical studies of the structural glasses.8) It would be interesting to create a phenomenological model with the rugged free-energy landscape picture be able to reproduce violation of the fluctuation-dissipation theorem in order to understand the physical meanings of the violation factor $X\left(t, t^{\prime}\right)$. (1)

\section{Acknowledgements}

I wish to acknowledge valuable discussions with Takashi Odagaki, Akira Yoshimori and Jun Matsui.

\section{Appendix: derivation of the steady-state value of the out-of-phase susceptibility}

Here, we evaluate the steady-state value of the out-of-phase susceptibility. We start with the expression of the susceptibility $\chi(\omega, t)$ in terms of the response function $R\left(t, t^{\prime}\right)$;

$$
\chi(\omega, t)=\int_{0}^{t} d t^{\prime} R\left(t, t^{\prime}\right) \exp \left[i \omega\left(t-t^{\prime}\right)\right] .
$$

Noting that the asymptotic form of the correlation function is given in eq. (3.5) and the response function is given by the time derivative of the correlation function, i.e. $-\partial_{t} C\left(t, t^{\prime}\right) / k_{B} T$, when the dynamics for the REM is trap type, i.e. eq. (2.2), (6) we can evaluate the steady-state value of the susceptibility from eq. (A-1).

At first, we divide the right hand side of eq. (A-1) into two parts as

$$
\chi(\omega, t)=\int_{0}^{t-t^{1-b}} d t^{\prime} R\left(t, t^{\prime}\right) e^{i \omega\left(t-t^{\prime}\right)}+\int_{t-t^{1-b}}^{t} d t^{\prime} R\left(t, t^{\prime}\right) e^{i \omega\left(t-t^{\prime}\right)},
$$

where we assume $0<b<1$. By using the relation $k_{B} T R\left(t, t^{\prime}\right)=-\partial_{t} C\left(t, t^{\prime}\right)$ and the asymptotic form of the correlation function, eq. (3.5), we can evaluate the first term as

$$
\text { |the first term } \mid \leq \frac{2 A \alpha}{k_{B} T}\left[t^{2 \alpha(b-1)}-t^{-2 \alpha-b}\right] .
$$

Hence, the first term becomes 0 in the long time limit, $t \rightarrow \infty$. Next, we evaluate the second term. Since $t-t^{\prime} \ll t^{\prime}$ in the region of the integration, the time translational invariance holds, i.e. $R\left(t, t^{\prime}\right)$ 
is the function of the time difference $t-t^{\prime}$. Rewriting the response function as $R\left(t-t^{\prime}\right)$, the second term is given as

$$
\text { the second term }=\int_{t-t^{1-b}}^{t} d t^{\prime} R\left(t-t^{\prime}\right) e^{i \omega\left(t-t^{\prime}\right)}=\int_{0}^{t^{1-b}} d \tau R(\tau) e^{i \omega \tau} .
$$

Hence, the second term becomes in the long time limit $\int_{0}^{\infty} d \tau R(\tau) e^{i \omega \tau}$. Gathering these results, the out-of-phase susceptibility is given in the long time limit as

$$
\lim _{t \rightarrow \infty} \chi^{\prime \prime}(\omega, t)=\int_{0}^{\infty} d \tau R(\tau) \sin (\omega \tau)=-\frac{1}{k_{B} T} \int_{0}^{\infty} d \tau \frac{d C(\tau)}{d \tau} \sin (\omega \tau)
$$

Then, noting that the decay of the correlation function $C(\tau)$ is faster than $1 / \tau$ when $\alpha>1 / 2$ and is slower than $1 / \tau$ when $\alpha<1 / 2$, by using the asymptotic form of the correlation function eq. (3.5) we reach the expression eq. (4.4).

[1] H. Frauenfelder, S. G. Sligar and P. G. Wolynes: Science 254 (1991) 1598.

[2] For a review, see E. Vincent, H. Hammann, M. Ocio, J.-P. Bouchaud and L. F. Cugliandolo: in Proceedings of the Sitges Conference on Glassy systems, edited by E. Rubi (Springer, Berlin, 1996); cond-mat/9607224.

[3] F. Lefloch, J. Hammann, M. Ocio and E. Vincent, Europhys. Lett. 18 (1992) 647; J. O. Andersson, J. Mattsson and P. Nordblad, Phys. Rev. B 48 (1993) 1397; E. Vincent, J.-P. Bouchaud, J. Hammann and F. Lefloch, Phil. Mag. B 71 (1995) 489.

[4] K. Jonason, E. Vincent, J. Hammann, J.-P. Bouchaud and P. Nordblad, Phys. Rev. Lett. 81, (1998) 3243.

[5] J.-P. Bouchaud and D. S. Dean, J. Phys. I France 5 (1995) 265; M. Sasaki and K. Nemoto, J. Phys. Soc. Jpn. 69 (2000) 2283 ; cond-mat/0010443.

[6] M. Sasaki and K. Nemoto, J. Phys. Soc. Jpn. 69 (2000) 3045.

[7] L. F. Cugliandolo and J. Kurchan, Phys. Rev. Lett. 71 (1993) 173; J. Phys. A 27 (1994) 5749; L. F. Cugliandolo, J. Kurchan and L. Peliti, Phys. Rev. E 55 (1997) 3898.

[8] G. Parisi, Phys. Rev. Lett. 79 (1997) 3660; J. -L. Barrat and W. Kob, Europhys. Lett. 46 (1999) 637.

[9] There are some surveys in this direction. For example, L. F. Cugliandolo, J. Kurchan and G. Parisi, J. Phys. I 4 (1994) 1641; L. Laloux and P. L. Doussal, Phys. Rev. E 57 (1998) 6296. 